\title{
MOVILIDAD DE ESTUDIANTES UNIVERSITARIOS EN IBEROAMÉRICA: INSTRUMENTOS PARA EL RECONOCIMIENTO DE LOS ESTUDIOS
}

\author{
Manuel Jaén García (*), Ana Madarro Racki (**)
}

SÍNTESIS: La puesta en marcha del Programa de Intercambio y M ovilidad Académica (PIMA) entre universidades iberoamericanas, hizo necesario el diseño de estrategias y de instrumentos válidos para todas las entidades educativas componentes de las redes, que permitiesen el reconocimiento académico de forma automática de los estudios cursados en la universidad de destino por la de origen.

Dadas las enormes diferencias existentes en los sistemas universitarios, en los planes de estudio, e incluso en los calendarios académicos entre las universidades integrantes de las redes, fue preciso un amplio estudio de campo, que culminó con el diseño de una metodología cuyos pilares fundamentales son la confianza entre las instituciones, la transparencia informativa, la reciprocidad y la flexibilidad. Todo ello ha permitido una gran fluidez en el intercambio informativo y el funcionamiento de las redes con eficacia.

SÍNTESE: A promoção do Programa de Intercâmbio e Mobilidade Acadêmica (PIMA) entre universidades ibero-americanas tornou necessário o desenho de estratégias e de instrumentos válidos para todas as entidades educativas componentes das redes, que permitissem o reconhecimento acadêmico de forma automática dos estudos cursados na universidade de destino pela a de origem.

Dadas as enormes diferenças existentes nos sistemas universitários, nos planos de estudo, e inclusive nos calendários acadêmicos entre as universidades integrantes das redes, foi preciso um amplo estudo de campo, que culminou com o desenho de uma metodologia cujos pilares fundamentais são a confiança entre as instituições, a transparência informativa, a reciprocidade e a flexibilidade, tudo o que permitiu uma grande fluidez no intercâmbio informativo e o funcionamento das redes com eficiência.

(*) Catedrático de Economía Aplicada y vicerrector de Relaciones Internacionales de la Universidad de Almería, España.

$(* *)$ Coordinadora de programas de la OEI. 


\section{INTRODUCCIÓN}

El objeto de este articulo es reseñar la experiencia realizada en redes del Programa de Intercambio y Movilidad Académica (PIMA) para desarrollar, de forma adecuada, el reconocimiento académico de los estudios cursados en una institución del programa por estudios pertenecientes a otra universidad de la red.

En la actualidad no existe en Iberoamérica un sistema de comparabilidad y de reconocimiento de estudios análogo al European Credit Transfer System (ECTS), desarrollado en Ia Unión Europea como consecuencia de la experiencia del programa de movilidad estudiantil Sócrates-Erasmus, en el que se produce el reconocimiento de modo automático. La larga experiencia del programa de movilidad ha permitido poner a punto instrumentos muy perfeccionados.

Desde el inicio del diseño del PIMA se tomó en cuenta esta carencia de modelos consensuados y comunes a la región que permitiese de forma clara y sencilla el reconocimiento, pero se estipuló como condición de viabilidad del programa asegurar que la movilidad de los estudiantes fuera acompañada del correspondiente reconocimiento de los estudios cursados. Las universidades adoptaron una nítida decisión en este sentido, al tiempo que reconocieron la diversidad institucional en la región, lo que requirió un desarrollo progresivo de criterios que aportara transparencia y fructificara en propuestas metodológicas para el reconocimiento de los estudios.

Para los coordinadores (institucional y académico) se planteó desde el primer momento la necesidad de trabajar sobre las dificultades que implicaba el proyecto, por la necesidad de obtener acuerdos entre instituciones universitarias muy diferentes. No obstante, se llegó a la puesta en común de una serie de criterios y de documentos a través de los que plasmarlos, que se definen en las siguientes páginas.

\section{METODOLOGÍA}

Para la Organización de Estados Iberoamericanos (OEI), el reconocimiento de estudios es la condición fundamental del PIMA, y la forma de garantizarlo ha sido la tarea central dada al grupo de trabajo inicial para diseñar el programa y para poner en marcha la experiencia 
piloto. Inicialmente este objetivo se fue alcanzando mediante una estrategia que privilegiaba la confianza mutua entre las universidades asociadas en red y la autoridad de los equipos de gobierno de las que participaban, como expresión de una voluntad política a favor de la cooperación interuniversitaria en la región, que se materializó en la firma de acuerdos al más alto nivel de las instituciones, que garantizan al estudiante el reconocimiento del período de estudios cursados en la universidad de destino a través del PIMA. Para ello se diseñaron los instrumentos y los procedimientos que recogen estos compromisos: compromiso previo de reconocimiento de estudios, contrato de estudios.

Sin embargo, el desarrollo del PIMA, así como las tendencias actuales en el campo de la educación superior, exigieron seguir avanzando en el desarrollo conceptual de estrategias, de criterios y de instrumentos que permitieran intercambiar información entre las universidades, y comparar los planes y los sistemas de estudio. Se trataba, entonces, de avanzar en la transparencia informativa y en los procedimientos de comparación. Por eso, en la convocatoria del PIMA correspondiente al año 2002 , se pidió a las universidades que, en la presentación de sus proyectos de movilidad de estudiantes, explicaran la forma en la que organizarían la tarea de intercambio y de análisis de planes de estudio de las áreas temáticas en las que realizaría dicha movilidad.

Al mismo tiempo, la OEI se propuso potenciar los recursos metodológicos del dispositivo de trabajo en red de las universidades que participaron en la experiencia piloto del PIMA, para desarrollar elementos conceptuales del programa aún poco definidos en la región: formulas de comparabilidad entre los sistemas, y estudios que facilitaran la movilidad con reconocimiento de estudios.

Para ello, el modelo metodológico del proyecto se basó en grupos de trabajo por áreas temáticas o redes PIMA, que analizaron y sistematizaron criterios y modal idades de comparabilidad traducibles en un producto final, que se ha utilizado como marco de referencia en las siguientes ediciones del PIMA.

\section{EL PROGRAMA PIMA: REALIDAD Y PROYECCIÓN DE FUTURO}

El Programa de Intercambio y Movilidad Académica entre universidades iberoamericanas surge como iniciativa de la OEI en 1999. 
Tras la fase de diseño, se organiza como experiencia piloto en el curso 2000-2001, con el ánimo de cubrir una necesidad notoriamente detectada en el ámbito de la cooperación entre los países del área. Hasta ese momento, el único procedimiento que permitía la movilidad de estudiantes, sólo de forma bilateral entre España y Latinoamérica, era el Programa de Cooperación Interuniversitaria (PCl). Pese a su importancia y a la buena acogida de esta iniciativa, el programa, desde sus inicios, se caracterizó por la corta duración de la estancia, sólo dos meses, y por la falta de reconocimiento de los estudios realizados. Eso convertía la estancia de los estudiantes en universidades de otros países en una pasantía, que aprovechaba el período vacacional producido por la diferencia entre los calendarios académicos.

EI PIMA busca algo más. En primer lugar, es un programa multilateral organizado en redes temáticas, en las que pueden participar universidades españolas, portuguesas y de América Latina. En segundo lugar, se organiza de modo que los estudiantes puedan obtener provecho académico de su trabajo, pues consiguen el reconocimiento de los estudios cursados en la universidad de destino por la de origen. Este reconocimiento se realiza a través de los convenios firmados entre las universidades, la red y la OEI, y de los contratos de estudio suscritos por el estudiante y el coordinador institucional del PIMA en la universidad de origen.

EI PIMA presenta como aspectos diferenciales con otros programas su carácter multilateral e iberoamericano, y que se dirige al nivel de pregrado de los estudios universitarios. Su estructura conceptual se desarrolla sobre los siguientes pilares: el valor de la movilidad de los estudiantes, el trabajo en red, la focalización en áreas temáticas, y el reconocimiento de los estudios.

A partir del curso 2001-2002, la consolidación del PIMA se convierte en un hecho reconocido en el ámbito al que se dirige. Aunque se han propuesto diversas iniciativas paralelas, como las recomendaciones del documento de la XI Cumbre de J efes de Estado y de Gobierno preparado por la Secretaría de Cooperación Ibercam ericana (SECIB $)^{1}, 0$ los acuerdos firmados entre la Conferencia de Rectores de las Universidades Españolas (CRUE) y sus homólogas de México (ANUIES), Brasil (ANDIFES), Colombia (ASCUN) y Argentina (CIN), ninguna de ellas se ha

\footnotetext{
${ }^{1}$ Análisis y potencialidades de la cooperación iberoamericana en educación superior.
} 
concretado en un programa semejante. En este sentido, la Comisión de América Latina de la Sectorial de Relaciones Internacionales de la CRUE (CEURI), en su reunión del 20 de julio de 2001 , y después de considerar la necesidad de promover un programa de movilidad estudiantil de carácter multilateral, general, amplio, con reconocimiento académico, con financiación adecuada y duración suficiente, estimó necesaria la creación de un órgano internacional coordinador, recomendando que fuera la OEI quien asumiera ese papel. Por su parte, el ya citado documento de la SECIB propuso que en el Programa Iberoamericano de Movilidad de Estudiantes de Educación Superior, en caso de ser aprobado como Programa Cumbre, se subsumiera y se absorbiera el PIMA.

En consecuencia, dada la experiencia, la satisfacción de los participantes y la estructura organizativa desarrollada, parece deseable y conveniente un crecimiento sostenido y sostenible del programa, es decir, la extensión y ampliación del PIMA bajo la coordinación de la OEI.

\section{PROGRAMAS ALTERNATIVOS}

Si bien existen algunas experiencias e iniciativas de movilidad de estudiantes a nivel subregional o de intercambios bilaterales que evidencian el interés estratégico que el tema presenta para la cooperación universitaria, hoy no hay ningún programa iberoamericano alternativo al PIMA, aunque sí un amplio consenso en cuanto a la necesidad de proyectos de este tipo. En el documento de laSECIB elaborado con motivo de la XI Cumbre se dice textualmente: «La movilidad internacional de estudiantes constituye una modalidad de cooperación que está actualmente muy considerada y que figura en la agenda de casi todos los programas de cooperación en educación superior [...] Actualmente existen algunas iniciativas y programas de fomento de la movilidad de estudiantes entre países iberoamericanos que, aunque todavía a pequeña escala, permiten identificar las limitaciones prácticas que supone actualmente esta modalidad de cooperación. Una de las iniciativas en marcha está promovida por la Organización de Estados I beroamericanos y ofrece un marco adecuado para la movilidad de estudiantes de pregrado».

En el mismo sentido, como quedó dicho, se manifestó la Comisión de América Latina del CEURI. En las conclusiones de la reunión celebrada el 20 de julio de 2001, se consideró prioritaria la necesidad de un programa de cooperación: «Todas las universidades coinciden en 
la necesidad y oportunidad de establecer un programa de cooperación interuniversitario iberoamericano, capaz de fortalecer los sistemas de educación superior de los países y de avanzar en la construcción de un espacio latinoamericano de Educación Superior, promoviendo la convergencia de los diferentes sistemas educativos». Más adelante se abogó por la necesidad de una institución coordinadora del programa de cooperación, recom endándose que fuera laOEI el organismo que asumiera esa función.

De igual forma se expresaron los vicerrectores y los directores de relaciones internacionales de las universidades iberoamericanas en las conclusiones de la reunión cel ebrada los días 13 y 14 de junio de 2001. En cuanto a los ámbitos y a las modalidades de la cooperación, se propuso: «2. La cooperación en la formación de pregrado, mediante el fomento de la movilidad estudiantil con pleno reconocimiento académico, aprovechando la experiencia positiva del programa Erasmus de las universidades europeas, y mediante la creación de proyectos académicos compartidos y de iniciativas de armonización de currículos académicos».

Por último, en la Declaración de Lima sobre Cooperación Universitaria Iberoamericana se sugirió la creación de un programa iberoamericano de cooperación interuniversitaria, que, entre otros propósitos, debe «prestar igualmente atención preferente a la movilidad estudiantil y docente, por la experiencia formativa que ella implica y por su contribución a la creación del espacio académico iberoamericano; en tal sentido se deberán incrementar las becas y ayudas que promuevan dicha movilidad».

Por tanto, parece ser precisa la creación de un programa de este tipo a gran escala, o, como se propone aquí, la ampliación del PIMA, máxime cuando la propia Unión Europea ha puesto en marcha el programa Erasmus-Mundus para estudiantes de tercer ciclo, que busca la ampliación de este plan a todos los países del mundo.

\section{ESTRATEGIAS E INSTRUMENTOS ADECUADOS PARA EL RECONOCIMIENTO DE ESTUDIOS}

Para la realización de este proyecto se partió de un documento base que fue puesto a disposición de todas las universidades participan- 
tes en la red. Dicho documento se utilizó como punto de arranque para los talleres celebrados por las diferentes redes.

En tres de los talleres participó de forma directa el coordinador académico del proyecto, obteniendo información concreta de los trabajos realizados por los demás. Tales talleres correspondieron a las redes PIMA de Desarrollo Agrario y Economía, red PIMA-Auditoría, y red Agroalimentaria de Escala.

Los objetivos de los talleres fueron dos:

- Desarrollar modelos e instrumentos de comparabilidad entre estudios, que permitieran su reconocimiento en los programas de intercambio y movilidad adaptados a las características de los programas de estudios ofertados en las universidades de la red.

- Sistematizar una metodología de trabajo para el conjunto del PIMA, y sentar las bases para la progresiva comparabilidad de los sistemas de educación superior con vistas a la integración regional.

La metodología de trabajo fue la siguiente:

- Presentación y discusión del documento base.

- Presentación de los sistemas educativos de los países participantes, con especial incidencia en la educación superior.

- Descripción de la estructura, de la organización y de los estudios de cada universidad, sobre todo del área temática objeto del trabajo.

- Análisis de los diferentes calendarios académicos, teniendo en cuenta las diferencias hemisféricas.

- Análisis de los diferentes métodos de evaluación y calificación en las universidades participantes en la red.

- Examen comparativo de dichos métodos, buscando elementos comunes destinados al reconocimiento académico de los estudios cursados en otras universidades, considerando para ello las diferentes metodologías de reconocimiento académico. 
- Puesta en común de un método de reconocimiento

- Elaboración del modelo y de la propuesta de documento

- Análisis y acuerdos organizativos para la realización de las movilidades y de los servicios de apoyo a los estudiantes de intercambio.

Debe destacarse que, además, otras redes PIMA celebraron reuniones preparatorias y de coordinación previas al inicio del intercambio de estudiantes. En sus reuniones siguieron una estructura de trabajo semejante, y aportaron los resultados de su trabajo a la coordinación institucional del PIMA en la OEI. Las redes PIMA que desarrollaron esos encuentros fueron: IBEROECO y Ciencias de la Tierra, ambas coordinadas por la Universidad de Barcelona (España), y celebradas en La Habana; INSIDE, coordinada por la U niversidad Nacional del Litoral (Argentina), y celebrada en Guatemala; Humboldt-Historia y RIEDE, coordinadas por la Universidad Pablo de Olavide (España), y celebradas en Lima.

Así mismo, coordinadores del PIMA en diversas universidades y la OEI, han participado en seminarios regionales y subregionales sobre movilidad de estudiantes, intercambiando experiencias sobre la cuestión del reconocimiento con responsables de otros programas similares de ámbito subregional, como, por ejemplo, el Seminario Internacional sobre Reconocimiento Académico en la Movilidad Estudiantil, organizado por la REDCIUN (responsable de la cooperación internacional de las universidades argentinas), ycelebrado en las U niversidades Nacional deCatamarca, Nacional de Córdoba y Nacional del Litoral, todas de Argentina.

En el documento base se analizan las diferentes formas en las que se puede realizar el reconocimiento.

Los contenidos, a partir de los propuestos inicialmente, y después del trabajo realizado en los talleres y en diferentes seminarios y con el material documental consultado, pueden organizarse en dos ejes temáticos:

- Criterios metodológicos para realizar la comparabilidad entre los estudios.

- Criterios metodológicos para alcanzar la transparencia informativa. 


\section{CRITERIOS METODOLÓGICOS PARA REALIZAR LA COMPARABILIDAD ENTRE LOS ESTUDIOS}

Los principios sobre los que se basa el intercambio de estudiantes son los siguientes:

- Confianza entre las instituciones: el conocimiento mutuo entre las instituciones docentes y la fiabilidad en la calidad de la enseñanza impartida, constituyen el punto de partida para la movilidad de estudiantes.

- Transparencia informativa: las instituciones firmantes de los acuerdos deben tener un amplio conocimiento de los planes de estudio, de los calendarios académicos, de los programas de las materias a cursar, de la organización de la docencia, de los sistemas de calificación y de sus posibles equivalencias, y de los planes de trabajo de las universidades contrapartes. En un epígrafe posterior se desarrolla un modelo de guía informativa que resulta adecuado para dar cumplida respuesta a esta transparencia.

- Reciprocidad: el acuerdo entre las instituciones debe ser simétrico y contemplar los mismos derechos y deberes para los estudiantes de una y de otra, y las mismas obligaciones de las universidades hacia los estudiantes. Para cualesquiera efectos, y durante su permanencia en el aula, el alumno de movilidad debe ser considerado como un miembro más de la universidad de acogida.

- Flexibilidad: las diferencias organizativas en cuanto a planes de estudio, a sistemas de calificación, a métodos de superación de las materias cursadas por los estudiantes, etc. son muy elevadas, aun entre las universidades de un mismo país. La situación es todavía más complicada cuando se trata de universidades de diferentes países, en las que, incluso, hasta el calendario académico es distinto. Por ello, es necesario aplicar la máxima flexibilidad posible a los reconocimientos académicos que se realicen entre diferentes universidades. La flexibilidad debe llevar consigo el rigor en la interpretación del trabajo de reconocimiento, pero, en ningún caso, la rigidez. La flexibilidad debe formar parte del espíritu de todos los acuerdos de reconocimiento que se firmen, y debe inculcarse no sólo a los coordinadores 
institucionales o académicos del PIMA, sino a los decanos y a los profesores que estén implicados en mayor o menor medida en las materias que sean objeto de reconocimiento en las redes.

Estos principios generales son reconocidos como necesarios por la mayoría de los responsables de la educación superior en la región, pero en muchos casos hay coincidencia en diagnosticar características de rigidez en los sistemas curriculares de las universidades latinoamericanas y europeas, incompatibilidad en los despliegues académicos (asignaturas anuales y semestrales) y en los cal endarios lectivos, y rigidez o celo en el personal docente. Todo ello, al impedir una articulación académica entre las universidades, y junto con dificultades en la gestión administrativa de estos asuntos en la propia universidad, entorpece considerablemente la movilidad de estudiantes, que se ve agravada por elementos externos que obstaculizan la libre circulación de personas en la región².

\section{SISTEMAS DE RECONOCIMIENTO: VENTAJAS E INCONVENIENTES}

El reconocimiento académico no es ni supone en ningún caso una convalidación. El concepto de convalidación implica la práctica igualdad de los programas de una determinada materia o asignatura cursada en dos universidades distintas. La convalidación se realiza por el profesor de la asignatura, con el refrendo del jefe del área o director del departamento, en su caso. La única calificación de la convalidación es «aprobado».

Dadas las diferencias entre los programas cursados en materias análogas en distintas universidades del mismo o de diversos países, resulta utópico hablar de convalidación en el contexto en el que nos movemos.

El concepto de reconocimiento académico es, al mismo tiempo, más flexible y más realista. Si partimos de la base de la calidad análoga de los estudios realizados en diferentes universidades para obtener el mismo titulo, debemos concluir que no es imprescindible que el alumno curse una determinada asignatura o un programa específico para obtener una óptima formación.

\footnotetext{
${ }^{2}$ Por ejemplo, la exigencia de visados o la de portar una cantidad mínima de dinero para entrar en algunos países.
} 
En última instancia, sería factible -y hasta útil para su formación-, que alumnos de universidades del país A cursasen asignaturas entroncadas con la historia o con la economía del país $B$, y les fuese reconocida en su universidad de origen, como materia cursada, la historia o la economía de su propio país.

En términos más concretos, sería posible realizar el reconocimiento con cuatro metodologías diferentes:

- Por asignaturas: este método supone el análisis materia por materia de los planes de estudio de las titulaciones de las diferentes universidades que van a realizar los intercambios. Si se quiere realizar un examen con detenimiento, será necesario estudiar los programas de las distintas asignaturas. La máxima rigidez implicaría reconocer sólo aquellas asignaturas con programas iguales, o con semejanzas en un $80 \%$ on un $90 \%$ de sus contenidos. Un sistema más flexible consideraría reconocibles asignaturas de nombres iguales o equival entes, y realizaría el reconocimiento en esos casos. Lógicamente, es factible cualquier tipo de solución intermedia.

Este sistema tiene como ventaja la aceptación casi mayoritaria del profesorado y de los departamentos afectados, así como la implicación del profesorado, de las áreas, de los departamentos y de la facultad en el proceso de comparación de los planes de estudio con la suficiente reflexión que trae consigo, y con la necesidad de conocer la realidad universitaria de otros países.

- Por créditos o por horas lectivas: este sistema supone tan solo reconocer en la universidad de origen el número de créditos (por ejemplo, en España, con el actual sistema, cada crédito equivale a diez horas lectivas) ${ }^{3}$ cursados en la universidad de destino, sin tener en cuenta las materias 0 asignaturas concretas.

\footnotetext{
${ }^{3}$ El Real Decreto 1125/2003 establece el concepto de crédito adaptado al espacio europeo de educación superior en los siguientes términos: unidad de medida del haber académico que representa la cantidad de trabajo del estudiante para cumplir los objetivos del programa de estudios, y que se obtiene por la superación de cada una de las materias que integran los planes de estudios de las diversas enseñanzas conducentes a la obtención de títulos universitarios de carácter oficial y con validez en todo el territorio
} 
La mayor ventaja del sistema es su enorme sencillez y flexibilidad, pues el alumno y el coordinador académico pueden decidir qué asignaturas van a ser reconocidas en la universidad de origen, sin tener en cuenta los criterios del profesorado o del área correspondiente.

El sistema implica una gran confianza entre las universidades yen la labor del coordinador académico, al que se supone sensato y buen conocedor de la titulación que coordina.

El principal inconveniente deriva de las reticencias que puede provocar en el profesorado de las diferentes asignaturas o materias el reconocimiento de las suyas sin su previo conocimiento.

Este sistema es el que, en mayor o menor medida, se aplica en las universidades europeas dentro del programa SócratesErasmus, y en el programa Séneca de las universidades españolas.

- Tablas de equivalencias: es un sistema intermedio entre los ateriores. Seiniciaconlaelabaración, parpantedelas áreas odepartam entosy la aprobaciónpor la juntadegobiernode la facultado porel órgano que correspanda, deunatabla de equivalencias, en laque, utilizandb lam áxim aflexibilidadposible, se reflejan las m aterias de la universidad de destino que serían reconocibles en la de origen.

Iatablackequivalencias facilitaengranm edich la laborch los coordinadores acadén icos, yaqueatom atizasutrabajo en la realización del contrato de estuctios. Ista renlización inplica atods los estam entos yelim ina aalquier tipo de reticenciadel profesoradb, pues hacolaboradbyhacladosu aprobaciónalos reconocim ientosprevistosenell laporm edio de la junta de centro o de gobiemo.

Susprincipales inconvenientes son la lentitudensuelaboraciónporel largoprocesoburocráticoquetraeconsigo, yla posible rigidez por la intransigencia que puedh tener una parte del profescrado.

nacional. En esta unidad de medida se integran las enseñanzas teóricas y prácticas, así como otras actividades académicas dirigidas, con inclusión de las horas de estudio y de trabajo que el estudiante debe realizar para alcanzar los objetivos formativos propios de cada una de las materias del plan de estudios correspondiente. 
En algunas universidades españolas se está realizando este proceso como una segunda fase del reconocimiento académico por créditos cursados.

- Por bloques semestrales o anuales: dadas las diferencias en cuanto al número de horas lectivas semestrales o anuales en las distintas universidades, así como la disparidad en cuanto a la cantidad de materias o asignaturas a cursar, puede resultar factible e incluso recomendable que el reconocimiento se realice por bloques completos.

Este sistema es aún más flexible que el de créditos, porque no implica ningún trabajo previo discriminatorio del coordinador académico y porque facilita mucho la labor del alumno, ya que éste se puede matricular en aquellas materias que corresponden al semestre que va a cursar, sin ningún otro trámite.

Los problemas que plantea saltan a la vista, pues dadas las diferencias en los planes de estudio, el alumno puede volver a cursar asignaturas, puede cursar más o menos materias, 0 tener más o menos horas lectivas que en su universidad de origen. Hay que emplear un sistema arbitrario, al igual que en el reconocimiento por créditos aunque empeorado, al aplicar una determinada calificación a las asignaturas cursadas.

El inconveniente por el lado del profesorado estaría en su posible irritación por no poder intervenir en la calificación de las asignaturas que imparte.

Este sistema se utiliza en la doble titulación de universidades europeas.

Las diferentes redes han aplicado metodologías ligeramente distintas, basadas en el reconocimiento por asignaturas. En todo momento ha primado el principio de flexibilidad y la búsqueda de beneficio para el estudiante. En el acta de la reunión de la red Desarrollo agrario y económico, se puede leer: «Los miembros de la red [...] entienden asimismo que es necesario para su futuro ad optar un criterio general para el reconocimiento [...] (en la actualidad) entienden como lo más adecuado el reconocimiento por asignaturas, comprometiéndose a la mayor amplitud de criterio y utilizando cada una el procedimiento más adecuado y acorde a la normativa interna de cada institución». 
Por su parte, en el acta de la reunión de la red PIMA-Auditoria, celebrada en Lima del 5 al 7 de marzo de 2002, se lee: «Las materias 0 cursos aprobados en las universidades de destino serán reconocidos en su integridad en las universidades de origen bajo el criterio de reconocimiento por asignaturas, dentro de una política de amplia flexibilidad y transparencia, aplicando equivalencias o compensación según sea el caso».

El criterio de equivalencias implica que existen materias en la universidad de destino que son equivalentes (en un elevado porcentaje) a las que se pueden cursar en la de origen; mediante el segundo criterio, compensación, se pueden reconocer como materias recibidas en la universidad de origen otras seguidas en la de destino que no tienen una clara correspondencia. Fundamentalmente, se considera que la flexibilidad y los beneficios que obtiene el estudiante por su conocimiento de otra realidad social y académica son superiores a los costes de no cursar una determinada materia que podrá estudiar en cursos de postgrado con posterioridad, o bien por sí mismo.

En cuanto a la reunión de la red Agroalimentaria de Escala, en su acta se dice «[...] la propuesta que finalmente podría ser viable de aplicación en estos sistemas universitarios, sería una combinación de dos o más modalidades a discutir con mayor profundidad».

De las reuniones mantenidas con representantes de otras redes se deduce que, de manera genérica, todas aplican en mayor o menor medida un sistema de reconocimiento por asignaturas con gran flexibilidad en cuanto a dicho reconocimiento, y siempre, salvo en casos muy excepcionales, buscando el beneficio del estudiante.

No está unificado el procedimiento del reconocimiento, que depende de la estructura interna de cada universidad, aunque por lo general existe alguien, llámese vicerrector 0 director de relaciones internacionales, decano, director o jefe de carrera, que es el coordinador institucional y el máximo responsable del reconocimiento, cuyo criterio prima por encima del de los profesores.

En todas las reuniones se constató la necesidad de crear una nueva conciencia, un cambio mental en el profesorado de todas las universidades, sobre todo en el de las latinoamericanas, para poder llevar a cabo de forma generalizada el intercambio. La actitud obstruccionista de algunos profesores suele impedir una adecuada fluidez en los inter- 
cambios. Por ello, es necesario conseguir que docentes y discentes estimen análoga la calidad de la docencia en todas las universidades, que el profesorado se considere similar, y que los programas impartidos en otras universidades se vean como equivalentes a los de la suya. De esa forma, se podrá realizar de manera completa y adecuada un programa de movilidad. Para conseguirlo, es fundamental el trabajo de coordinadores institucionales y académicos, que, a través de seminarios y de reuniones, puedan crear el espíritu apropiado en su profesorado y en los estudiantes. En ese sentido, es importante que se transmitan a la comunidad académica en su conjunto las ideas esenciales que subyacen tras el PIMA:

- La insistencia en la movilidad como un valor en sí mismo.

- La necesidad de contrastar y de comparar la calidad de los sistemas educativos.

- La búsqueda de espacios comunes de conocimiento y de comprensión.

- El conocimiento de otras gentes, e incluso de otros idiomas (casos de Brasil y Portugal).

- El futuro de las titulaciones en Iberoamérica, con homologaciones automáticas de un país a otro.

- En definitiva, y como resumen de todo lo anterior, la creación de un espacio iberoamericano de educación superior.

\section{TRANSPARENCIA EN LA INFORMACIÓN: GUÍAS INFORMATIVAS}

Para la correcta aplicación y puesta en marcha del PIMA en las diferentes universidades y redes, es necesario poder disponer de documentos que puedan aportar información de manera clara y concisa sobre la universidad y sobre lo que la rodea.

La guía informativa es el instrumento que proporciona transparencia al programa, y es de utilidad práctica pues responde, en un lenguaje llano y comprensible, a todas y cada una de las preguntas que pueden formularse profesores y alumnos de las universidades tanto de origen como de posible destino. En ese sentido y desde esa perspectiva, debe organizarse su contenido y cada una de sus secciones. Dado que los idiomas de las universidades iberoamericanas son el español y el 
portugués, sus ediciones deberían ser bilingües. Además, sería recomendable que dichas ediciones fueran electrónicas, divulgando por la red las direcciones de acceso, gracias a su mayor versatilidad y facilidad a la hora de realizar cambios y correcciones, y, cómo no, a su menor coste.

La transparencia en la información implica el conocimiento exhaustivo, tanto por parte de los estudiantes como de los coordinadores, de las estructuras de los planes de estudio, de los programas de las asignaturas, del profesorado, de los criterios de evaluación, del número de créditos, de las modalidades de examen, etc., de las universidades que forman parte de la red. Para ello, y de forma homogénea, es necesario que las diferentes universidades del programa elaboren guías informativas. En estas guías se deberán reflejar los siguientes puntos:

- Descripción general de la ciudad en la que está situada la universidad: localización geográfica, climatología, accesos, medios de transporte, alojamientos para estudiantes, centros culturales y de diversión, restaurantes, etc.

- Descripción de la universidad: plano de su emplazamiento, servicios que presta, tales como comedores, colegios mayores, bibliotecas, instalaciones deportivas, conexiones a Internet, etc., así como medios de transporte para el acceso a la universidad.

Centros que componen la universidad y titulaciones que se imparten en cada uno de ellos. Requisitos de acceso a cada titulación.

Trámites burocráticos que tiene que afrontar el estudiante de intercambio, y unidades administrativas a las que tiene que dirigirse, incluyendo nombres de los responsables, direcciones y teléfonos.

- Otras cuestiones de interés.

- Guías informativas por titulaciones, en las que se incluirían: el plan de estudios de cada titulación, con el número de créditos o de horas lectivas por asignatura; el calendario académico, y una ficha por materia en la que se especificaría el objetivo de la ésta, su temario resumido, el profesorado que la imparte, el método de enseñanza y el procedimiento de examen. 


\section{CONCLUSIONES}

\subsection{NECESIDAD DEL PIMA}

El recorrido realizado durante el período 2000-2004 ha puesto de manifiesto el amplio consenso existente entre las universidades componentes de las redes PIMA, en cuanto a la necesidad del programa y a la voluntad manifiesta de buscar fórmulas que permitan su ampliación a todas las universidades de los países implicados.

Sin embargo, dicha ampliación no debería desvirtuar el sentido último del programa en lo que se refiere a sus aspectos de intercambio de alumnos con reconocimiento de estudios realizados, y, en un futuro próximo, también de docentes, como vía para la puesta en marcha de un espacio iberoamericano de educación superior, que con tanto ahínco se persigue. El programa es, por otra parte, una oportunidad única para que los gobiernos y las universidades iberoamericanas puedan buscar una cooperación adecuada, que permita contrarrestar la fuerte influencia del modelo anglosajón.

\subsection{UN ÚNICO PROGRAMA DE MOVILIDAD PARA IBEROAMÉRICA}

Se deberían aunar los esfuerzos y las iniciativas que se están produciendo desde diferentes instancias para poner en marcha programas de intercambio estudiantil con reconocimiento académico subsumiéndolos en el PIMA, pues se trata de un programa ya rodado, con experiencia, creíble, y en el que tienen fe las universidades que participan. El modelo, en el que las universidades han contribuido activamente con la OEI para la elaboración de las bases de participación, de los términos de referencia, de los procedimientos del programa, e igualmente en los procesos de selección, es muy satisfactorio, pues une la transparencia y la participación activa de los actores implicados en el proceso. Esta concepción de trabajo garantiza la credibilidad de los programas de movilidad de los estudiantes.

\subsection{ESTRATEGIAS E INSTRUMENTOS DE COMPARABILIDAD ENTRE ESTUDIOS}

El desarrollo futuro del programa supondrá, cada vez más, la utilización de sistemas de reconocimiento a través de créditos y de horas 
lectivas, y la realización por parte de todas las universidades involucradas de guías informativas de calidad que permitan a los interesados una amplia comprensión de los requisitos y de las facilidades que tendrán para su participación con garantía de éxito en el programa. La manera más versátil y económica de guía es la que se presenta mediante formato electrónico.

\subsection{INSTRUMENTOS DE COMUNICACIÓN ENTRE LA COORDINACIÓN DEL PROGRAMA Y LOS ESTUDIANTES}

Por otro lado, y de forma complementaria, una herramienta útil para la coordinación central del PIMA sería la creación de una revista electrónica, en la que los estudiantes pudiesen volcar sus experiencias, a la vez que tener acceso a las novedades que se pudieran introducir en el programa. El formato de esa revista podría ser diseñado por la coordinación del programa, en colaboración con una comisión de las universidades participantes.

Entre los espacios de la revista digital podría incluirse un lugar común para el intercambio de opiniones en tiempo real, con una denominación que sería algo así como «el foro», «el ágora» o «la plaza» del PIMA. Se trataría de un espacio de conversación gratuito, en el que los estudiantes tendrían oportunidad de intercambiar experiencias e informaciones, o de poner en conocimiento de los coordinadores los problemas con los que se pueden encontrar. 


\title{
Contactar
}

Revista lberoamericana de Educación

\author{
Principal OEI
}

